\title{
Production of Interspecific Hybrids between Hydrangea macrophylla and Hydrangea arborescens via Ovary Culture
}

Ming Cai ${ }^{1}$, Ke Wang, Le Luo, Hui-tang Pan, and Qi-xiang Zhang Beijing Key Laboratory of Ornamental Plants Germplasm Innovation \& Molecular Breeding, National Engineering Research Center for Floriculture, Beijing Laboratory of Urban and Rural Ecological Environment and College of Landscape Architecture, Beijing Forestry University, Beijing 100083, China

\author{
Yu-yong Yang \\ Kunming Yang Chinese Rose Gardening Co., Ltd., Kunming 650500, China
}

Additional index words. bigleaf Hydrangea, interspecific hybridization, SSRs, smooth hydrangea, ovule culture

\begin{abstract}
Hydrangea macrophylla is the most popular species in the genus Hydrangea because of its large and brightly colored inflorescences. Since the early 1900s, numerous cultivars with showy flowers have been selected. Although many $H$. macrophylla cultivars have been developed, cold hardiness is still the major limitation to their outdoor use. Hydrangea arborescens is a small attractive shrub or subshrub native to northeastern parts of the United States, which is valued for its hardiness. Interspecific breeding of $\boldsymbol{H}$. arborescens and $\boldsymbol{H}$. macrophylla has been tried, but putative hybrid seedlings either died at an early stage or were not verified. We made successful hybridizations between $H$. macrophylla 'Blue Diamond' and $\boldsymbol{H}$. arborescens 'Annabelle' and used in vitro ovary culture to produce viable plants. Hybrids were intermediate in appearance between parents, but variable in leaves, inflorescences, and flower color. The success of this hybridization was confirmed by six simple sequence repeat (SSR) genetic markers. The maternal chromosome number was 36 , and the paternal number was 38 . Chromosome counts of hybrids indicated that nearly half of them were aneuploids. Male fertility of progeny was evaluated by fluorescein diacetate staining of pollen. Twelve out of 14 hybrids $(85.7 \%)$ had male fertility. We documented the first flowering progeny of $H$. macrophylla and $H$. arborescens, suggesting an effective beginning to a cold hardiness breeding program.
\end{abstract}

The genus Hydrangea is one of the most popular ornamental flowers because of its large and showy inflorescences. The genus comprises at least 30 taxa with centers of diversity in eastern Asia, eastern North America, and South America (Kardos et al., 2009; McClintock, 1957). This genus is further divided into two sections: Hydrangea and Cornidia. Section Cornidia consists of climbing species from tropical and subtropical areas and sect. Hydrangea contains mainly temperate species that are ornamentally attractive and commonly cultivated (Kudo et al., 2008).

Bigleaf hydrangea (H. macrophylla) is the most commonly cultivated member of the Hydrangeaceae family and is native to southern

Received for publication 9 July 2015. Accepted for publication 25 Oct. 2015.

This study was supported by the Fundamental Research Funds for the Central Universities (YX2013-05) and Beijing Higher Education Young Elite Teacher Project (YETP0743).

${ }^{1}$ Corresponding author. E-mail: jasoncai82@163. com.

HortScience Vol. 50(12) December 2015

China and Japan (McClintock, 1957; 1923). The species is cultivated as a cut flower and flowering pot plant. It is valued for its large and brightly colored inflorescences that range in color from blue to pink, unchangeably or depending on the amount of aluminum in the soil (Reed et al., 2001, 2008; Takeda et al., 1985). However, its use in areas colder than USDA hardiness zone 6 is limited by the lack of cold hardiness in the flower buds, which are formed on the previous year's growth. Even in zones 6 and 7, late spring freezes may damage floral buds, resulting in greatly reduced flowering (Reed, 2000). Smooth hydrangea $(H$. arborescens $)$ is a shrub or subshrub (up to 2-3 $\mathrm{m}$ tall) native to eastern North America and is hardy to zone 4. It is valued for its attractive, delicate corymbs of white flowers in early summer (Dirr, 1998). 'Annabelle' is a cold-tolerant smooth hydrangea cultivar that can survive in Beijing (hardiness zone 7) and other cities in northern China (zones 6 and 7).

Hybridization between distantly related species has been used in ornamental crop breeding to move desirable genes from one taxon to another (Langton, 1987). Interspecific or even intergeneric hybridizations have been made successfully in Hydrangea. Some examples are $H$. arborescens $\times H$. involucrata, $H$. peruviana $\times H$. serratifolia, $H$. scandens ssp. chinensis $\times H$. macrophylla, H. macrophylla $\times H$. paniculata, $H$. macrophylla $\times H$. angustipetala, and H. macrophylla $\times$ Dichroa febrifuga (Jones and Reed 2006; Kardos et al., 2009; Kudo et al., 2008; Reed et al., 2001, 2008; van Gelderen and van Gelderen 2004). It seemed that seeds were produced more easily when $H$. macrophylla used as the maternal parent, which was also mentioned by Reed et al. (2001). Other interspecific crosses between $H$. macrophylla and $H$. arborescens or $H$. macrophylla and $H$. quercifolia failed because of postzygotic barriers. The putative hybrid seedlings died at the cotyledonary stage or the first set of true leaves stage (Reed, 2000). One effective approach to overcome postzygotic barriers that cause hydrangea seedling mortality is embryo rescue (Kudo et al., 2008). This technique has previously been used in efforts to hybridize $H$. macrophylla and $H$. arborescens. Putative hybrid plants were obtained from ovule cultures initiated from seedlings on cotyledonary stage, but the hybridity of these plants was not verified by a genetic analysis (Kudo and Niimi, 1999).

In an effort to increase the cold hardiness of $H$. macrophylla and expand the market into cold areas, $H$. arborescens was hybridized with $H$. macrophylla to combine the cold hardiness of $H$. arborescens with the wide spectrum of flower color present in H. macrophylla. The present study describes the production of interspecific hybrids between H. macrophylla 'Blue Diamond', 'Schneeball', and $H$. arborescens 'Annabelle' via ovule culture. The offspring were identified as hybrids using morphological, cytological, and SSR data.

\section{Materials and Methods}

Plant material and pollinations. Hydrangea macrophylla 'Blue Diamond', 'Schneeball', and $H$. arborescens 'Annabelle' were used in this study. To improve the success rate of hybridization, $H$. macrophylla was used as the maternal parent. $H$. arborescens 'Annabelle', the male parent, was collected from Beijing Botanical Garden (Beijing, China). The parental plants and the hybrids were grown in the nursery of Kunming Yang Chinese Rose Gardening Co., Ltd. (Kunming, China) following the method described by Jones and Reed (2006). Crosses were made during the Summers of 2012 and 2013.

Ovary culture. Capsules containing fertilized ovaries were collected $60-65 \mathrm{~d}$ after pollination. The capsules were washed for $2 \mathrm{~h}$ with tap water, then disinfected in $70 \%$ ethanol for $30 \mathrm{~s}$, and then immersed in $0.1 \%$ $(\mathrm{w} / \mathrm{v})$ mercuric chloride for $8 \mathrm{~min}$, followed by $4-5$ rinses with sterile distilled water. After disinfection, enlarged ovaries were 


\begin{tabular}{|c|c|c|c|c|c|c|c|}
\hline \multirow[b]{2}{*}{ Hybridization } & \multirow[b]{2}{*}{$\begin{array}{c}\text { Flowers } \\
\text { pollinated (no.) }\end{array}$} & \multicolumn{3}{|c|}{ Seed germination ${ }^{z}$} & \multicolumn{3}{|c|}{ Ovary culture ${ }^{y}$} \\
\hline & & $\begin{array}{c}\text { Fruits } \\
\text { harvested (no.) }\end{array}$ & $\begin{array}{c}\text { Seeds } \\
\text { germinated (no.) }\end{array}$ & $\begin{array}{c}\text { Seedling } \\
\text { survival (no.) }\end{array}$ & $\begin{array}{c}\text { Ovaries } \\
\text { cultured (no.) }\end{array}$ & $\begin{array}{c}\text { Plants } \\
\text { obtained (no.) }\end{array}$ & Survival (no.) \\
\hline $\begin{array}{l}\text { H. macrophylla } \\
\text { 'Schneeball' } \times \\
\text { H. arborescens 'Annabelle' }\end{array}$ & 1030 & 248 & 0 & - & 83 & 12 & 0 \\
\hline $\begin{array}{l}\text { H. macrophylla } \\
\text { 'Blue Diamond' } \times \\
\text { H. arborescens 'Annabelle' }\end{array}$ & 1210 & 307 & 18 & 0 & 195 & 56 & 14 \\
\hline
\end{tabular}

${ }^{\mathrm{z} F r u i t}$ allowed to mature on plant. Seeds were sown on soilless media according to Reed (2000).

${ }^{\mathrm{y}}$ Ovaries harvested 60-65 d after pollination and cultured on $1 / 2$ Murashige and Skoog media $+1.0 \mathrm{mg} \cdot \mathrm{L}^{-1} 6-\mathrm{benzylaminopurine}+1.0 \mathrm{mg} \cdot \mathrm{L}^{-1} 2-$ naphthaleneacetic acid. The number of seedlings surviving is defined as the number of plants living after being transferred from aseptic conditions.

placed directly on half-strength Murashige and Skoog (MS) media (Murashige and Skoog, 1962), supplemented with 2\% sucrose to promote maturation. Six different combinations of 6-benzylaminopurine (6BA; 0.5, 1.0, $2.0 \mathrm{mg} \cdot \mathrm{L}^{-1}$ ) and 2-naphthaleneacetic acid (NAA; $0.1,1.0 \mathrm{mg} \cdot \mathrm{L}^{-1}$ ) were added to the media to test the most effective recipe for induction. Each medium contained 30 ovaries as three repeats. The media were adjusted to $5.8-6.0 \mathrm{pH}$ with $1 \mathrm{M} \mathrm{NaOH}$ before autoclaving at $121^{\circ} \mathrm{C}$ for $20 \mathrm{~min}$. The selected medium was used to culture capsules of 'Schneeball' $\times$ 'Annabelle' and 'Blue Diamond' $x$ 'Annabelle' for viable hybrid seedlings. Calli induced in ovaries were abandoned to eliminate the interference of somatic cells. Seedlings were subcultured every 3-4 weeks onto fresh medium of $1 / 2$ MS media without hormones. All cultures were incubated at $25 \pm 2{ }^{\circ} \mathrm{C}$ under a 16 -h photoperiod $(80$ $\left.\mu \mathrm{mol} \cdot \mathrm{m}^{-2} \cdot \mathrm{s}^{-1}\right)$. After rooting, the plants were transplanted into plastic pots containing a mixture of peatmoss and perlite in a ratio of $3: 1(\mathrm{v} / \mathrm{v})$. The plants were acclimatized for 1 month and then cultivated in a greenhouse under the same conditions as the parental plants.

Morphological comparisons. Rooted cuttings were prepared in Summer 2013 based on the protocol of Jones and Reed (2006). Morphology of the leaves and inflorescences were measured on parents and flowering hybrid individuals in Summer 2014 following the methods of Reed et al. (2008).

Mitotic cytology. Root tips were immersed in $2 \mathrm{~mm}$ 8-hydroxyquinoline for $2 \mathrm{~h}$ at room temperature $\left(22^{\circ} \mathrm{C}\right)$, then rinsed in distilled water, fixed in a solution of ethanol: acetic acid $(3: 1)$ for $24 \mathrm{~h}$ at $4{ }^{\circ} \mathrm{C}$, finally transferred to $70 \%$ ethanol at $-20{ }^{\circ} \mathrm{C}$ until needed. Before examination, root tips were hydrolyzed in $1 \mathrm{M} \mathrm{HCl}$ at $55^{\circ} \mathrm{C}$ for $12 \mathrm{~min}$, rinsed with distilled water, and soaked in $1 \%$ aceto-orcein. The meristematic region of the root tip was squashed in aceto-orcein, and chromosomes were counted. Ten metaphase cells were examined.

SSR analysis. To verify the success of the hybrid cross, 14 putative hybrids and parents were compared using six SSR loci (STAB111_112, STAB125_126, STAB309_310, STAB321_322, STAB391_392, and STAB501_502) that have been shown to produce polymorphic data in
Hydrangea (Rinehart et al., 2006). Fresh, new leaves of the 14 putative hybrids and parents were collected and dried in silica-gel dessicant. Deoxyribonucleic acid (DNA) was extracted from leaf tissues following the protocol of the Plant Genomic DNA Kit (Tiangen, Beijing) and quantified using a NanoDrop Spectrophotometer 2000 (Thermo Fisher Scientific, Waltham, MA). Polymerase chain reaction amplification and SSR genotyping followed the protocol of Cai et al. (2011).

Pollen viability. Pollen viability was assessed according to Kardos et al. (2009). Three fields of 100 pollen grains each were counted per hybrid and parent and the mean number of fluorescent grains calculated for each genotype.

Statistical analysis. Excel (Microsoft Office, 2010) was used to analyze the data. Analysis of variance (F test, $P \leq 0.05$ ) and Duncan's multiple comparison test $(\alpha=0.05)$ were conducted with SPSS 17.0 software (SPSS Inc., Chicago, IL) to analyze significance. For the genetic identification analyses, a pairwise genetic similarity matrix based on SSR data from parents and offspring was estimated and principal-coordinate analysis
(PCoA) was performed using NTSYS 2.02a software.

\section{Results and Discussion}

Interspecific hybridizations. About 2200 crosses were made during 2012 and 2013. Nearly 550 mature fruits were harvested for sowing (Table 1). Viable seeds were collected from both of the interspecific hybridizations, but only the seeds of 'Blue Diamond' $x$ 'Annabelle' germinated. No obvious disease or nutritional problem was found that would explain the germination failure of 'Schneeball' $\times$ 'Annabelle'. The genus Hydrangea is composed of two sections, Hydrangea and Cornidia, which could be further subdivided into eight subsections. Interspecific breeding programs have crossed members of five subsections, from which, it could be concluded that postzygotic rather than prezygotic problems were the barriers of compatibility. In some intersubsection breeding experiments, ovaries expanded after controlled pollination, which indicated that there might be no prefertilization barriers (Jones and Reed, 2006; Kudo and Niimi, 1999; Kudo et al., 2008; Reed,

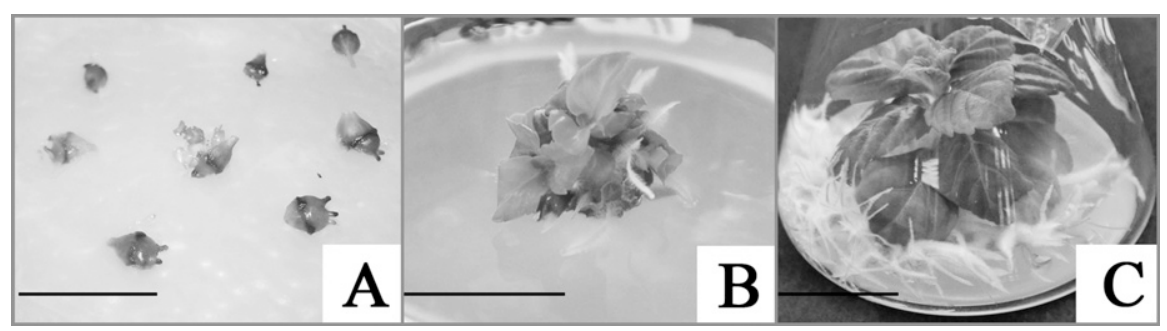

Fig. 1. Ovary culture procedure of Hydrangea macrophylla 'Blue Diamond' $\times$ Hydrangea arborescens 'Annabelle'. (A) Ovules mature and germinate 30-45 d after inoculation; (B) Seedlings are transferred to fresh medium and root; (C) Seedlings mature and are transferred to the greenhouse. Bar $=2.0 \mathrm{~cm}$.

Table 2. Effects of six hormone combinations on ovary maturation and seed germination.

\begin{tabular}{|c|c|c|c|c|}
\hline Basal media ${ }^{z}$ & 6-BA $\left(\mathrm{mg} \cdot \mathrm{L}^{-1}\right)$ & $\mathrm{NAA}\left(\mathrm{mg} \cdot \mathrm{L}^{-1}\right)$ & Inoculated ovaries (no.) & Germination $(\%)^{\mathrm{yx}}$ \\
\hline $1 / 2 \mathrm{MS}$ & 0.5 & 0.1 & 30 & $3.3 \pm 5.8 \mathrm{c}$ \\
\hline $1 / 2 \mathrm{MS}$ & 0.5 & 1.0 & 30 & $7.9 \pm 6.9 b$ \\
\hline $1 / 2 \mathrm{MS}$ & 1.0 & 0.1 & 30 & $8.3 \pm 2.7 b$ \\
\hline $1 / 2 \mathrm{MS}$ & 1.0 & 1.0 & 30 & $17.0 \pm 5.1 \mathrm{a}$ \\
\hline $1 / 2 \mathrm{MS}$ & 2.0 & 0.1 & 30 & $7.4 \pm 3.6 \mathrm{~b}$ \\
\hline $1 / 2 \mathrm{MS}$ & 2.0 & 1.0 & 30 & $4.25 \pm 4.67 \mathrm{c}$ \\
\hline
\end{tabular}

${ }^{\mathrm{z}} \mathrm{MS}=$ Murashige and Skoog; 6-BA = 6-benzylaminopurine; NAA = 2-naphthaleneacetic acid.

y Values represent means \pm SE. Letters indicate the level of significance according to Duncan's multiple comparison test $(\alpha=0.05)$.

${ }^{\mathrm{x}} 60-65 \mathrm{~d}$ after pollination ovaries were placed on media until seeds matured and germinated. 


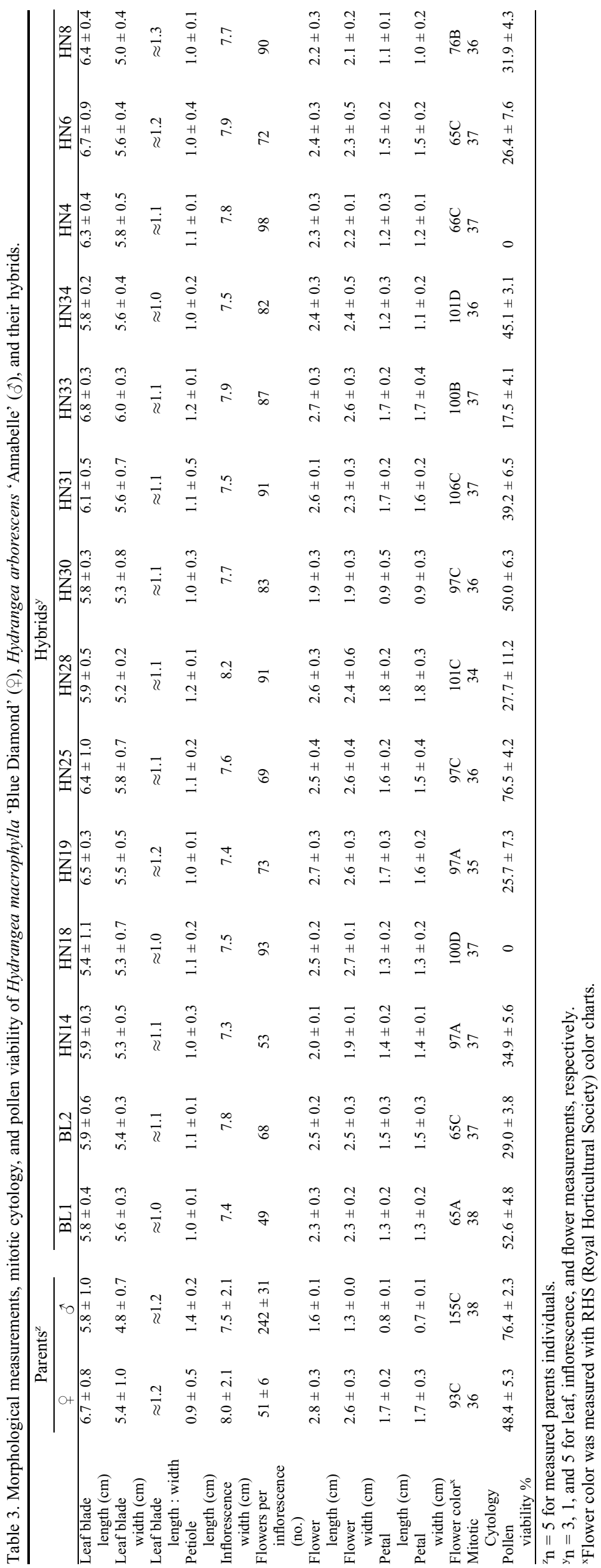

2000; Reed et al., 2001). Even in crosses of 'Blue Diamond' $x$ 'Annabelle', pollen tubes grew through the style to the base of the ovary (Kudo and Niimi, 1999). So, postzygotic barriers are the main cause of failure in Hydrangea interspecific crosses, usually resulting in seedless fruits or the early death of seedlings (Jones and Reed, 2006; Reed, 2000).

Increased numbers of fertile progeny have been observed when $H$. macrophylla is the maternal parent (Reed et al., 2001). In this study, $24.1 \%$ and $25.4 \%$ of the pollinated flowers of all interspecific crosses formed seed pods (Table 1). However, no seeds were produced when 'Schneeball' and 'Annabelle' were crossed. Only 3\% of fruits harvested from crosses of 'Blue Diamond' $x$ 'Annabelle' contained viable seeds. Sixteen seedlings died at the cotyledonary stage, while the remaining two seedlings died after the first set of true leaves emerged.

Ovary culture. In preliminary experiments, we compared excising the ovules and culturing the ovaries in the capsules. The excised ovules were too weak to ripen on the media, but the ovules cultured in situ matured and germinated. About 2 months after pollination, 180 enlarged ovaries that originated from additional crosses were harvested and placed upright on the MS medium surface to test the most suitable hormone combination. In the following 2-3 weeks, the ovaries turned gray and seemed to die. However, 30-45 d after inoculation, $\approx 3.3 \%$ to $17.0 \%$ of ovules ripened and germinated (Fig. 1). The most efficient medium for ovary culture and germination was $1 / 2 \mathrm{MS}$ with $1.0 \mathrm{mg} \cdot \mathrm{L}^{-1} 6$-BA and $1.0 \mathrm{mg} \cdot \mathrm{L}^{-1} \mathrm{NAA}$, which resulted in a significantly higher rate of ovary culture success $(17.0 \%$ germination; Table 2).

As shown in Table 1, 83 explants from 'Schneeball' $\times$ 'Annabelle' and 195 explants from 'Blue Diamond' $\times$ 'Annabelle' were cultured in the optimized medium. Nearly $14.5 \%$ of ovaries germinated from the cross of 'Schneeball' $\times$ 'Annabelle', but no seedlings survived when transferred to the greenhouse. Fifty-six ovaries germinated from the cross of 'Blue Diamond' $\times$ 'Annabelle', a quarter of which survived outdoors. Moreover, the 14 putative hybrids of 'Blue Diamond' $x$ 'Annabelle' flowered in the Summer of 2014.

The embryo rescue technique was necessary to hybridize the distantly related species $H$. macrophylla and $H$. arborescens (Kudo and Niimi, 1999). Success of the embryo rescue protocol relies on a variety of factors, including the choice of explants, culture medium, the addition of plant growth hormones, and incubation conditions (Khan et al., 2009; Molina et al., 2007; Tallón et al., 2013). Ovary culture is one possible embryo rescue method allowing ovules mature in situ, without being excised from the fruit. Ovary culture has also been successfully used to overcome postzygotic barriers in 
interspecific Lilium crosses (van Tuyl et al., 1991).

Morphological comparisons. Leaf blades of 'Annabelle' were shorter and considerably narrower than those of 'Blue Diamond' (Table 3). Mean leaf blade length and width were similar in the hybrids and 'Annabelle' (the male parent). The hybrids had leaf blade length-to-width ratios of $\approx 1.1$. The leaf blade length-to-width ratios of both parents were 1.2. The petioles of 'Blue Diamond' were noticeably shorter than those of 'Annabelle', while the hybrids measured were intermediate to the parents. It should be mentioned that the leaf texture of the 14 hybrids was more similar to 'Annabelle' (the paternal parent), in that they felt thinner and sparsely pubescent than the thick and glabrous texture of the leaves of 'Blue Diamond' (the maternal parent)

Mean inflorescence width was similar between parents and hybrids (Table 3 ). The 'Annabelle' inflorescences contained four to five times more flowers than the inflorescences of 'Blue Diamond'. The hybrids had a large number of flowers, which provides additional evidence of hybridity. Individual flower length and width were greater in 'Blue Diamond' than in 'Annabelle'. Mean flower and petal length or width of the hybrids was intermediate between the two parents (Table 3 ). The flower color of the hybrids was red (4) or blue (10), while the female parent was purple and the male parent was white in the same growing conditions (Fig. 2). The texture of the petals in the hybrids was close to the maternal parent.

Mitotic cytology. Examination of mitotic root tip cells revealed 38 chromosomes in H. arborescens 'Annabelle' (Fig. 3), which support the results of Kudo and Niimi (1999), who studied the same cultivar. For H. arborescens 'Dardom', the number was 38 as well (Jones and Reed, 2006). We observed 36 chromosomes in H. macrophylla 'Blue Diamond' (Fig. 3), which was consistent with previous studies of this species (Cerbah et al., 2001). The interspecific hybrids had 34-38 chromosomes, with nearly half of them (6/ 14) being 37 (Table 3). The odd number of chromosomes may result in sterility in flowering hybrids.

SSR analysis. Six polymorphic SSR markers were selected from Rinehart et al. (2006) to verify interspecific hybridity between parents and 14 progenies by comparing allele size variation. Two hybrids (BL1 and HN8) could be directly confirmed by SSR markers (STAB501_502) based on Mendelian expectations for gene segregation. The alleles of parents were $115 \mathrm{bp} / 145 \mathrm{bp}$ for the maternal parent and $154 \mathrm{bp} / 154 \mathrm{bp}$ for the paternal parent, while they were $115 \mathrm{bp} / 154 \mathrm{bp}$ in the progeny. Other putative hybrids had at least one allele from each parent or a recombination of parental SSR loci. The pairwise genetic similarity coefficient showed that the proportion of shared genetic variation between $H$. macrophylla and 14 hybrids varied from 0.43 to 0.87 and from 0.40 to 0.60 between hybrids and the paternal parent. Within hybrids, the similarity ranged from 0.47 to 0.93 . A three-dimensional plot of the PCoA showed clear separation between
$H$. arborescens (the paternal donor) and the offspring (Fig. 4). This may result from the influence of the maternal parent. The most genetic similarity observed between putative hybrids and $H$. macrophylla was 0.87 . This difference was most likely derived from the controlled pollination rather than somaclonal variation induced by tissue culture, for the data ranged from 0.980 to 0.983 (Similarity Coefficient) among 32 somaclonal samples of H. macrophylla (Liu et al., 2011). The molecular data provided evidence that the 14 plants obtained from the cross of 'Blue Diamond' $x$ 'Annabelle' were interspecific hybrids.

Pollen viability. Pollen viability was estimated in the hybrids and parents by fluorescein diacetate staining. 'Annabelle' and 'Blue Diamond' had $76.4 \%$ and $48.4 \%$ stainable pollen, respectively. Stainable

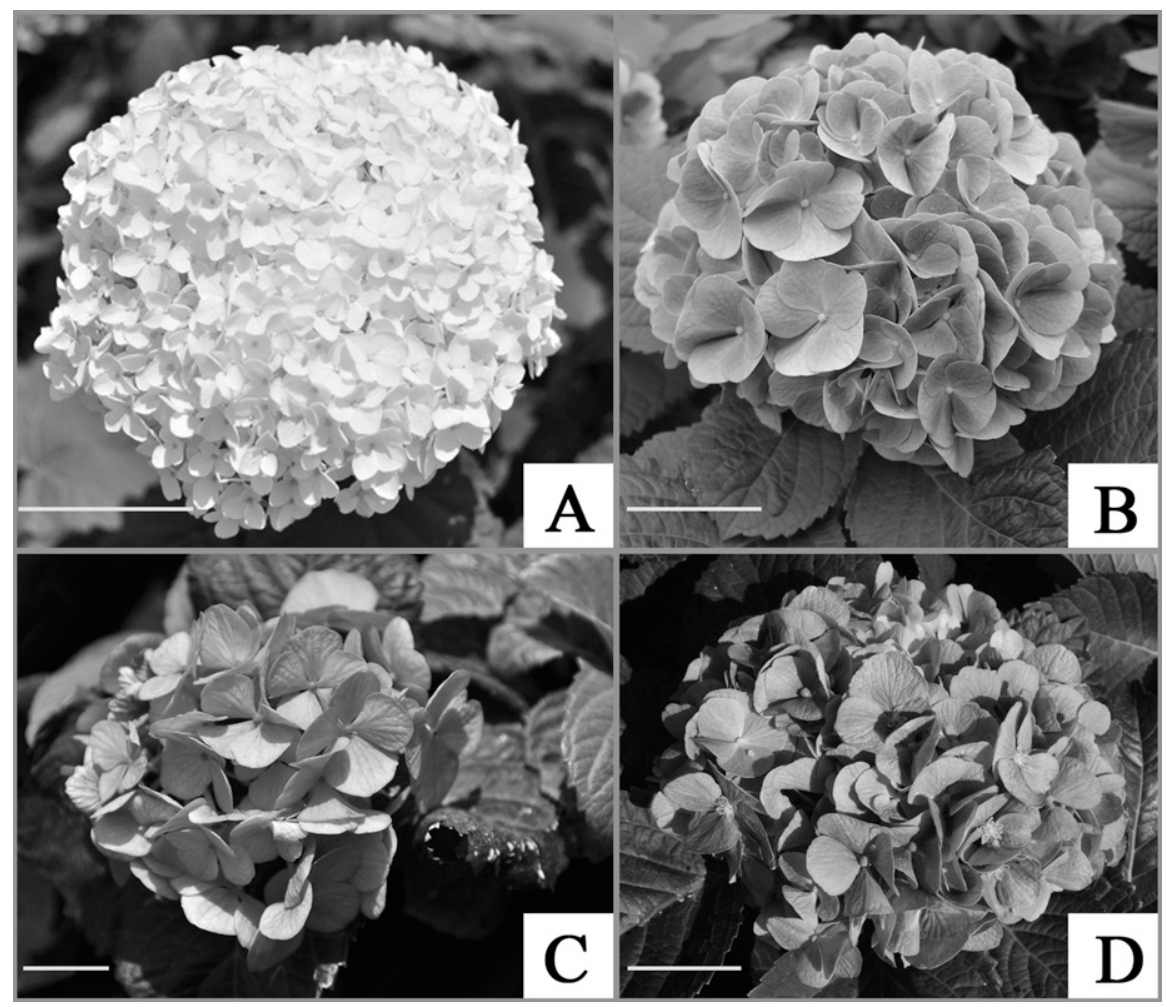

Fig. 2. Differences in flower color and morphology in hybrids and parents (A) Hydrangea arborescens 'Annabelle' with white flowers (RHS 155C). (B) Hydrangea macrophylla 'Blue Diamond' with violetblue flowers (RHS 93C). (C) 'Blue Diamond' $\times$ 'Annabelle' hybrid with red-purple flowers (RHS 65A). (D) 'Blue Diamond' $\times$ 'Annabelle' hybrid with purple flowers (RHS 76B). Bar $=2.0 \mathrm{~cm}$.

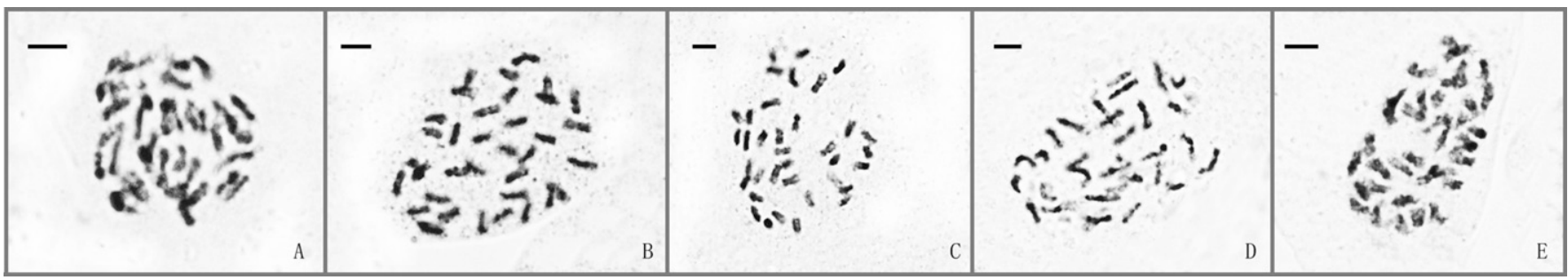

Fig. 3. Chromosomes of (A) Hydrangea arborescens 'Annabelle' $(2 \mathrm{n}=38)$, (B-D) Hydrangea macrophylla 'Blue Diamond' $\times$ H. arborescens 'Annabelle' hybrids $(2 \mathrm{n}=35,36,37$, respectively), $(\mathbf{E}) H$. macrophylla 'Blue Diamond' $(2 \mathrm{n}=36)$ from root tip cells. Bar $=4 \mu \mathrm{m}$. 


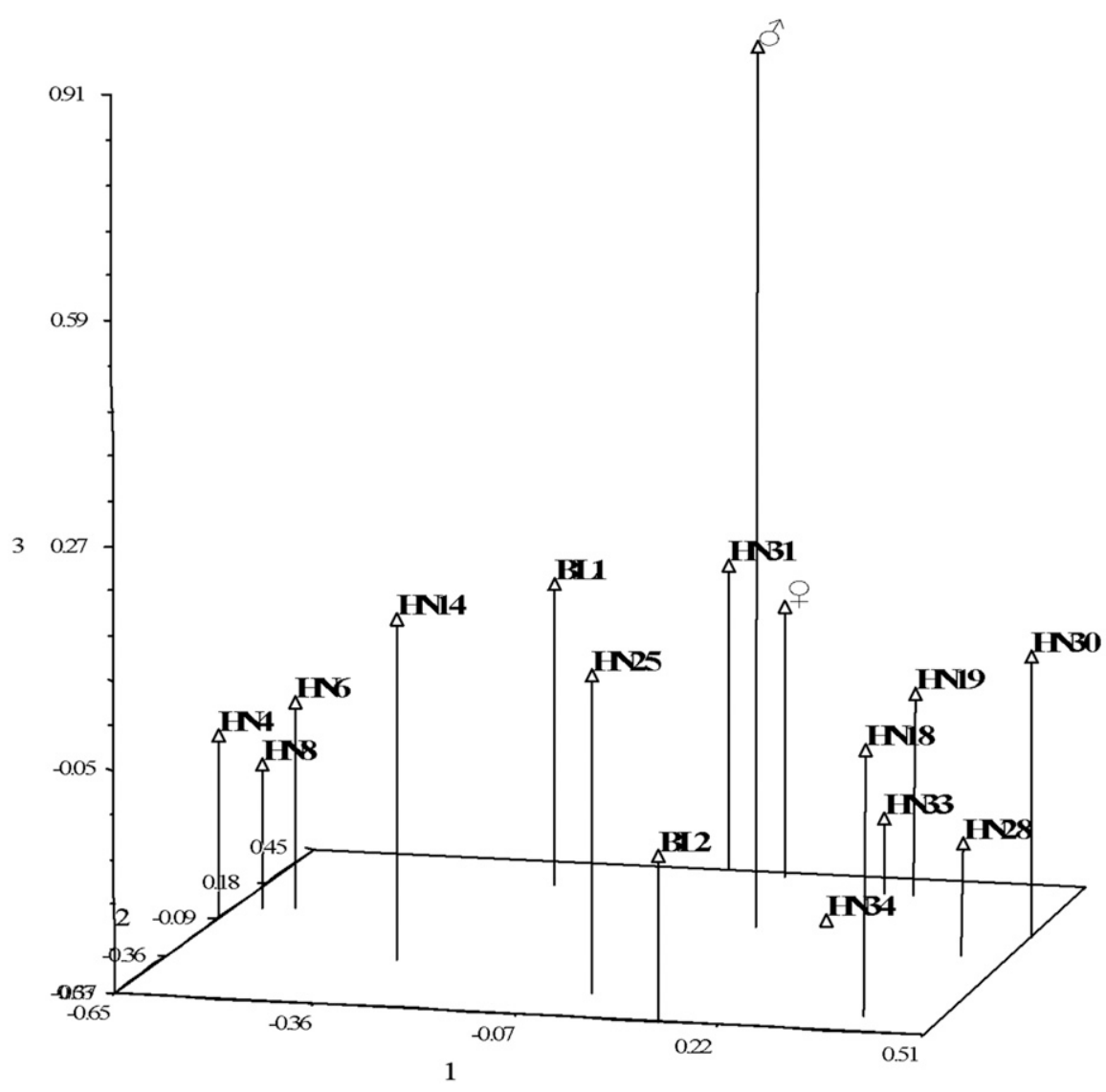

Fig. 4. A three-dimensional plot of the principal coordinate analysis (PCoA) based on data from six simple sequence repeat loci. + was Hydrangea macrophylla 'Blue Diamond'; $\hat{\jmath}$ was Hydrangea arborescens 'Annabelle'. BL and HN were the code names of interspecific hybrids.

pollen ranged from $0 \%$ to $76.5 \%$ among 14 hybrids (Table 3). In previous studies, pollen fertility as measured by stainable pollen of intergeneric hybrids between Dichroa febrifuga and $H$. macrophylla ranged from $0 \%$ to $73 \%$ (Kardos et al., 2009). No pollen was stained in HN4 and HN18 flowering plant, while HN25 had the highest rate of stained pollen, which might result from the 36 chromosomes. Not all hybrids with odd number of chromosomes appeared male-sterile, and female fertility still needs to be tested in future studies.

This study improves the methods to obtain flowering hybrids of $H$. macrophylla and $H$. arborescens. The success of the hybridization was verified by morphological comparisons, chromosome counts, and SSR analysis. Our results suggest it may be possible to increase the cold hardiness of cultivated hydrangeas using $H$. arborescens. The 14 hybrid individuals have been propagated and transplanted to some testing points in Beijing. They still need to be evaluated for cold hardiness, backcross fertility, and pest resistance. These hybrids will serve as the
Khan, E.U., X.Z. Fu, J. Wang, Q.J. Fan, X.S. Huang, G.N. Zhang, J. Shi, and J.H. Liu. 2009. Regeneration and characterization of plants derived from leaf in vitro culture of two sweet orange (Citrus sinensis (L.) Osbeck) cultivars. Sci. Hort. 120:70-76.

Kudo, N., T. Matsui, and T. Okada. 2008. A novel interspecific hybrid plant between Hydrangea scandens ssp. chinensis and H. macrophylla via ovule culture. Plant Biotechnol. 25:529-533.

Kudo, N. and Y. Niimi. 1999. Production of interspecific hybrids between Hydrangea macrophylla f. hortensia (Lam.) Rehd. and H. arborescens L. J. Jpn. Soc. Hort. Sci. 68:428439.

Langton, F.A. 1987. Breeding for improved ornamental plants. Academic Press, London.

Liu, F., L.L. Huang, Y. Li, P. Reinhoud, M.A. Jongsma, and C.Y. Wang. 2011. Shoot organogenesis in leaf explants of Hydrangea macrophylla 'Hyd1' and assessing genetic stability of regenerants using ISSR markers. Plant Cell Tissue Organ Cult. 104:111-117.

McClintock, E. 1957. A monograph of the genus Hydranega. Proc. Calif. Acad. Sci. 24:147256.

Molina, R.V., S. Castelló, A. García-Luis, and J.L. Guardiola. 2007. Light cytokinin interactions in shoot formation in epicotyl cuttings of Troyer citrange cultured. In vitro. Plant Cell Tissue Organ Cult. 89:131-140.

Murashige, T. and F. Skoog. 1962. A revised medium for rapid growth and bio-assays with tobacco tissue cultures. Physiol. Plant. 15:473497.

Reed, S.M. 2000. Compatibility studies in Hydrangea. J. Environ. Hort. 18:29-33.

Reed, S.M., D. Keri, K.D. Jones, and T.A. Rinehart. 2008. Production and characterization of intergeneric hybrids between Dichroa febrifuga and Hydrangea macrophylla. J. Amer. Soc. Hort. Sci. 133:89-91.

Reed, S.M., G.L. Riedel, and M.R. Pooler. 2001. Verification and establishment of Hydrangea macrophylla'Kardinal' $\mathrm{x} H$. paniculata 'Brussels Lace' interspecific hybrids. J. Environ. Hort. 19:85-88.

Rinehart, T.A., B.E. Scheffler, and S.M. Reed 2006. Genetic diversity estimates for the genus Hydrangea and development of a molecular key based on SSR. J. Amer. Soc. Hort. Sci. 131:787-797.

Takeda, K., M. Kariuda, and H. Itoi. 1985. Blueing of sepal colour of Hydrangea macrophylla. Phytochemistry 24:2251-2254.

Tallón, C.I., I. Porras, and O. Pérez-Tornero. 2013. High efficiency in vitro organogenesis from mature tissue explants of Citrus macrophylla and $C$. aurantium. In Vitro Cell. Dev. Biol. Plant 49:145-155.

van Gelderen, C.J. and D.M. van Gelderen. 2004. Encyclopedia of Hydrangeas. Timber Press, Portland, OR.

van Tuyl, J.M., M.P. Van Diën, M.G.M. van Kleinwee, J. Franken, and R.J. Bino. 1991. Application of in vitro pollination, ovary culture, ovule culture and embryo rescue for overcoming incongruity barriers in interspecific Lilium crosses. Plant Sci. 74:115-126.

Wilson, E.H. 1923. The hortensias: Hydrangea macrophylla DC and Hydrangea serrata DC. J. Arnold Arbor. 4:233-246. 